MISC-2010-01

\title{
QP-Structures of Degree 3 and 4D Topological Field Theory
}

\author{
Noriaki IKEDA ${ }^{1 *}$ and Kyousuke UCHINO2 ${ }^{\dagger}$ \\ 1 Maskawa Institute for Science and Culture, Kyoto Sangyo University, \\ Kyoto 603-8555, Japan \\ ${ }^{2}$ Department of Mathematics, Tokyo University of Science \\ Wakamiya 26, Shinjyuku, Tokyo, Japan
}

\begin{abstract}
A BV algebra and a QP-structure of degree 3 is formulated. A QP-structure of degree 3 gives rise to Lie algebroids up to homotopy and its algebraic and geometric structure is analyzed. A new algebroid is constructed, which derives a new topological field theory in 4 dimensions by the AKSZ construction.
\end{abstract}

*E-mail address: ikeda@yukawa.kyoto-u.ac.jp

${ }^{\dagger}$ E-mail address: k_uchino@oct.rikadai.jp 


\section{Introduction}

A BV algebra and a QP-structure has been motivated by the structure of the BatalinVilkovisky formalism of a gauge theory [1] and is its mathematical formulation [2]. In case of a topological field theory of Schwarz type, a BV formalism has been reformulated to the AKSZ formulation, which is a clear construction using geometry of a graded manifold [3] 4]. Application to higher $n+1$ dimensions has been formulated and new topological field theories in higher dimensions have been founded by applying this construction [5] [6] [7.

In $n=1$, a classical QP-structure is a Poisson structure on a manifold $M$ and is also a Lie algebroid on $T^{*} M$ from the explicit construction. This is equivalent to the construction of a Poisson structure by the Schouten-Nijenhuis bracket in a classical limit. The topological field theory in two dimensions constructed by the AKSZ formulation [4] is the Poisson sigma model [8] 9] and the quantization of this model on disc derives the Kontsevich formula of the deformation quantization on a Poisson manifold [11][12].

In $n=2$, a classical QP-structure is a Courant algebroid [13][14]. The topological field theory derived in three dimensions is the Courant sigma model [15] [16] [17].

However structures for higher $n$, more than 2, have not been understood enough apart from BF theories.

In this paper, we analyze $n=3$ case. A QP-structure of degree 3 leads us to a new type of algebroid, which is called a Lie algebroid up to homotopy. The notion of this algebroid is defined as a homotopy deformation of a Lie algebroid satisfying some integrability conditions. We will prove that a QP-structure of degree 3 on a N-manifold (nonnegatively graded manifold) is equivalent to a Lie algebroid up to homotopy. This QP-structure defines a new natural 4-dimensional topological field theory via the AKSZ construction.

The paper is organized as follows. In section 2, a BV algebra and a QP-structure of degree 3 are formulated. In section 3, a QP-structure of degree 3 is constructed and analyzed. In section 4, examples of QP-structures of degree 3 are listed. In section 5, the AKSZ construction of a topological field theory in four dimensions is formulated and examples are listed. $c$

${ }^{c}$ Very recently, Grützmann's paper appears which has overlaps with our paper [18]. 


\section{QP-manifolds and BV Algebras}

\subsection{Classical QP-manifold}

Definition 2.1 A graded manifold $\mathcal{M}$ is by definition a sheaf of a graded commutative algebra over an ordinary smooth manifold $M$.

In the following, we assume the degrees are nonnegative.

The structure sheaf of $\mathcal{M}$ is locally isomorphic to a graded commutative algebra $C^{\infty}(U) \otimes$ $S(V)$, where $U$ is an ordinary local chart of $M, S(V)$ is the polynomial algebra over $V$ and where $V:=\sum_{i \geq 1} V_{i}$ is a graded vector space such that the dimension of $V_{i}$ is finite for each $i$. For example, when $V=V_{1}, \mathcal{M}$ is a vector bundle whose fiber is $V_{1}^{*}$ : the dual space of $V_{1}$.

Definition 2.2 A graded manifold $(\mathcal{M}, \omega)$ equipped with a graded symplectic structure $\omega$ of degree $n$ is called a $\boldsymbol{P}$-manifold of degree $n$.

In the next section, we will study a concrete P-manifold of degree 3.

The structure sheaf $C^{\infty}(\mathcal{M})$ of a P-manifold becomes a graded Poisson algebra. The Poisson bracket is defined in the usual manner,

$$
\{F, G\}=(-1)^{|F|+1} \iota_{X_{F}} \iota_{X_{G}} \omega
$$

where $F, G \in C^{\infty}(\mathcal{M}),|F|$ is the degree of $F$ and $X_{F}:=\{F,-\}$ is the Hamiltonian vector field of $F$. We recall the basic properties of the Poisson bracket,

$$
\begin{aligned}
\{F, G\} & =-(-1)^{(|F|-n)(|G|-n)}\{G, F\}, \\
\{F, G H\} & =\{F, G\} H+(-1)^{(|F|-n)|G|} G\{F, H\}, \\
\{F,\{G, H\}\} & =\{\{F, G\}, H\}+(-1)^{(|F|-n)(|G|-n)}\{G,\{F, H\}\},
\end{aligned}
$$

where $n$ is the degree of the symplectic structure and $F, G, H \in C^{\infty}(\mathcal{M})$. We remark that the degree of the Poisson bracket is $-n$.

Definition 2.3 Let $(\mathcal{M}, \omega)$ be a P-manifold of degree $n$. A function $\Theta \in C^{\infty}(\mathcal{M})$ of degree $n+1$ is called a $\boldsymbol{Q}$-structure, if it is a solution of the classical master equation,

$$
\{\Theta, \Theta\}=0 \text {. }
$$

The triple $(\mathcal{M}, \omega, \Theta)$ is called a $\boldsymbol{Q P}$-manifold. 
We define an operator $Q:=\{\Theta,-\}$, which is called a homological vector field. From (2.2) we have the cocycle condition,

$$
Q^{2}=0
$$

which says that the homological vector field is a coboundary operator on $C^{\infty}(\mathcal{M})$ and defines a cohomology called the classical BRST cohomology.

\subsection{Quantum QP-manifold}

Definition 2.4 A graded manifold is called a quantum BV-algebra if it has an odd Laplace operator $\Delta$, which is a linear operator on $C^{\infty}(\mathcal{M})$ satisfying $\Delta^{2}=0$, and the graded Poisson bracket is given by

$$
\{F, G\}=(-1)^{|F|} \Delta(F G)-(-1)^{|F|} \Delta(F) G-F \Delta(G)
$$

where $F, G \in C^{\infty}(\mathcal{M})$.

If $n$ is odd, a P-manifold $(\mathcal{M}, \omega)$ has the odd Poisson bracket. If an odd P-manifold $(\mathcal{M}, \omega)$ has a volume form $\rho$, one can define an odd Laplace operator $\Delta$ (See [19]):

$$
\Delta F:=\frac{1}{2}(-1)^{|F|} \operatorname{div}_{\rho} X_{F}
$$

Here a divergence $\operatorname{div}_{\rho}$ is a map from a space of vector fields on $\mathcal{M}$ to $C^{\infty}(\mathcal{M})$ and is defined by

$$
\int_{\mathcal{M}} \operatorname{div}_{\rho} X F d v=-\int_{\mathcal{M}} X(F) d v,
$$

for a vector field $X$ on $\mathcal{M}$. The pair $(\mathcal{M}, \Delta)$ is called a quantum P-structure. An odd Laplace operator has degree $-n$.

Definition 2.5 A function $\Theta \in C^{\infty}(\mathcal{M})$ with the degree $n+1$ is called a quantum $Q$ structure, if it satisfies a quantum master equation

$$
\Delta\left(e^{\frac{i}{\hbar} \Theta}\right)=0
$$

where $\hbar$ is a formal parameter. The triple $(\mathcal{M}, \Delta, \Theta)$ is called a quantum QP-manifold. 
From the definition of an odd Laplace operator, the equation (2.4) is equivalent to

$$
\{\Theta, \Theta\}-2 i \hbar \Delta \Theta=0
$$

If we take the limit of $\hbar \rightarrow 0$ in (2.5), which is called a classical limit, the classical master equation $\{\Theta, \Theta\}=0$ is derived. Since $\Delta^{2}=0, \Delta$ is also a coboundary operator. This defines a quantum BRST cohomology. Let $\mathcal{O}^{\prime}=\mathcal{O} e^{\frac{i}{\hbar} \Theta} \in C^{\infty}(\mathcal{M})$ be a cocycle with respect to $\Delta$. The cocycle condition $\Delta\left(\mathcal{O}^{\prime}\right)=\Delta\left(\mathcal{O} e^{\frac{i}{\hbar} \Theta}\right)=0$ is equivalent to

$$
\{\Theta, \mathcal{O}\}-i \hbar \Delta \mathcal{O}=0
$$

The solutions of (2.6) are called observables in physics. In the classical limit, (2.6) is $\{\Theta, \mathcal{O}\}=$ $Q \mathcal{O}=0 . \mathcal{O}$ reduces to an element of a classical BRST cohomology.

\section{Structures and homotopy algebroids}

In this section, we construct and analyze a classical QP-structure of degree 3 explicitly.

\subsection{P-structures}

Let $E \rightarrow M$ be a vector bundle over an ordinary smooth manifold $M$. The shifted bundle $E[1] \rightarrow M$ is a graded manifold whose fiber space has the degree +1 . We consider the shifted cotangent bundle $\mathcal{M}:=T^{*}[3] E[1]$. It is a P-manifold of the degree 3 over $M$,

$$
T^{*}[3] E[1] \rightarrow \mathcal{M}_{2} \rightarrow E[1] \rightarrow M,
$$

where $\mathcal{M}_{2}$ is a certain graded manifold $\left.\right|^{d}$. The structure sheaf $C^{\infty}(\mathcal{M})$ of $\mathcal{M}$ is decomposed into the homogeneous subspaces,

$$
C^{\infty}(\mathcal{M})=\sum_{i \geq 0} C^{i}(\mathcal{M})
$$

where $C^{i}(\mathcal{M})$ is the space of functions of degree $i$. In particular, $C^{0}(\mathcal{M})=C^{\infty}(M)$ : the algebra of smooth functions on the base manifold and $C^{1}(\mathcal{M})=\Gamma E^{*}$ : the space of sections of the dual bundle of $E$.

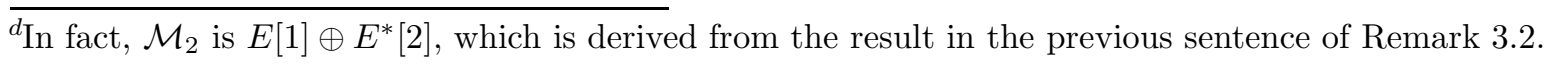


Let us denote by $(x, q, p, \xi)$ a canonical (Darboux) coordinate on $\mathcal{M}$, where $x$ is a smooth coordinate on $M, q$ is a fiber coordinate on $E[1] \rightarrow M,(\xi, p)$ is the momentum coordinate on $T^{*}[3] E[1]$ for $(x, q)$. The degrees of the variables $(x, q, p, \xi)$ are respectively $(0,1,2,3)$.

Two directions of counting the degree of functions on $T^{*}[3] E[1]$ are introduced. Roughly speaking, these are the fiber direction and the base direction.

Definition 3.1 (Bidegree, see also Remark 3.3 .3 in [14]) Consider a monomial $\xi^{i} p^{j} q^{k}$ on a local chart $(U ; x, q, p, \xi)$ of $\mathcal{M}$, of which the total degree is $3 i+2 j+k$. The bidegree of the monomial is, by definition, $(2(i+j), i+k)$.

This definition is invariant under the natural coordinate transformation,

$$
\begin{aligned}
x_{i}^{\prime} & =x_{i}^{\prime}\left(x_{1}, x_{2}, \ldots, x_{\operatorname{dim}(M)}\right) \\
q_{i}^{\prime} & =\sum_{j} t_{i j} q_{j}, \\
p_{i}^{\prime} & =\sum_{j} t_{i j}^{-1} p_{j}, \\
\xi_{i}^{\prime} & =\sum_{j} \frac{\partial x_{j}}{\partial x_{i}^{\prime}} \xi_{j}+\sum_{j k l}\left(\frac{\partial t_{j l}^{-1}}{\partial x_{i}^{\prime}} t_{l k}+\frac{\partial t_{k l}}{\partial x_{i}^{\prime}} t_{l j}^{-1}\right) p_{j} q_{k},
\end{aligned}
$$

where $t$ is a transition function. Since $T^{*}[3] E[1]$ is covered by the natural coordinates, the bidegree is globally well-defined (See also Remark 3.2 below.)

The space $C^{n}(\mathcal{M})$ is uniquely decomposed into the homogeneous subspaces with respect to the bidegree,

$$
C^{n}(\mathcal{M})=\sum_{2 i+j=n} C^{2 i, j}(\mathcal{M}) .
$$

Since $C^{2,0}(\mathcal{M})=\Gamma E$ and $C^{0,2}(\mathcal{M})=\Gamma \wedge^{2} E^{*}$, we have

$$
C^{2}(\mathcal{M})=\Gamma E \oplus \Gamma \wedge^{2} E^{*} .
$$

Remark 3.2 The P-manifold $T^{*}[3] E[1]$ is regarded as a shifted manifold of $T^{*}[2] E[1]$. The structure sheaf is also a shifted sheaf of the one on $T^{*}[2] E[1]$. In particular, the space $C^{2 i, j}$ is the shifted space of $C^{i, j}$ on $T^{*}[2] E[1]$. 
For the canonical coordinate on $\mathcal{M}$, the symplectic structure has the following form:

$$
\omega=\delta x^{i} \delta \xi_{i}+\delta q^{a} \delta p_{a}
$$

and the associated Poisson bracket has the following expression:

$$
\{F, G\}=F \frac{\overleftarrow{\partial}}{\partial x^{i}} \frac{\vec{\partial}}{\partial \xi_{i}} G-F \frac{\overleftarrow{\partial}}{\partial \xi_{i}} \frac{\vec{\partial}}{\partial x^{i}} G+F \frac{\overleftarrow{\partial}}{\partial q^{a}} \frac{\vec{\partial}}{\partial p_{a}} G-F \frac{\overleftarrow{\partial}}{\partial p_{a}} \frac{\vec{\partial}}{\partial q^{a}} G
$$

where $F, G \in C^{\infty}(\mathcal{M})$ and $\frac{\vec{\partial}}{\partial \phi}$ and $\frac{\overleftarrow{\partial}}{\partial \phi}$ are the right and left differentiations, respectively. Note that the degree of the symplectic structure is +3 and the one of the Poisson bracket is -3 . The bidegree of the Poisson bracket is $(-2,-1)$, that is,

$$
\{(2 i, j),(2 k, l)\}=(2(i+k)-2, j+l-1)
$$

where $(2 i, j) \ldots$ are functions with the bidgree $(2 i, j)$.

\subsection{Q-structures}

We consider a (classical) Q-structure, $\Theta$, on the P-manifold. It is required that $\Theta$ has degree 4. That is, $\Theta \in C^{4}(\mathcal{M})$. Because $C^{4}(\mathcal{M})=C^{4,0}(\mathcal{M}) \oplus C^{2,2}(\mathcal{M}) \oplus C^{0,4}(\mathcal{M})$, the Q-structure is uniquely decomposed into

$$
\Theta=\theta_{2}+\theta_{13}+\theta_{4}
$$

where the bidegrees of the substructures are $(4,0),(2,2)$ and $(0,4)$, respectively. In the canonical coordinate, $\Theta$ is the following polynomial:

$$
\Theta=f_{1{ }^{i} a}(x) \xi_{i} q^{a}+\frac{1}{2} f_{2}{ }^{a b}(x) p_{a} p_{b}+\frac{1}{2} f_{3}{ }^{a} b c(x) p_{a} q^{b} q^{c}+\frac{1}{4 !} f_{4 a b c d}(x) q^{a} q^{b} q^{c} q^{d},
$$

and the substructures are

$$
\begin{aligned}
\theta_{2} & =\frac{1}{2} f_{2}{ }^{a b}(x) p_{a} p_{b}, \\
\theta_{13} & =f_{1}{ }^{i}{ }_{a}(x) \xi_{i} q^{a}+\frac{1}{2} f_{3}{ }^{a}{ }_{b c}(x) p_{a} q^{b} q^{c}, \\
\theta_{4} & =\frac{1}{4 !} f_{4 a b c d}(x) q^{a} q^{b} q^{c} q^{d},
\end{aligned}
$$


where $f_{1}-f_{4}$ are structure functions on $M$. By counting the bidegree, one can easily prove that the classical master equation $\{\Theta, \Theta\}=0$ is equivalent to the following three identities:

$$
\begin{aligned}
\left\{\theta_{13}, \theta_{2}\right\} & =0, \\
\frac{1}{2}\left\{\theta_{13}, \theta_{13}\right\}+\left\{\theta_{2}, \theta_{4}\right\} & =0, \\
\left\{\theta_{13}, \theta_{4}\right\} & =0 .
\end{aligned}
$$

The conditions (3.8), (3.9) and (3.10) are equivalent to

$$
\begin{aligned}
& f_{1}{ }^{i}{ }_{b} f_{2}{ }^{b a}=0, \\
& f_{1}{ }^{k}{ }_{c} \frac{\partial f_{2}{ }^{a b}}{\partial x^{k}}+f_{2}{ }^{d a} f_{3}{ }^{b} c d+f_{2}{ }^{d b} f_{3}{ }^{a} c d=0, \\
& f_{1}{ }^{k}{ }_{b} \frac{\partial f_{1}{ }^{i} a}{\partial x^{k}}-f_{1}{ }^{k}{ }_{a} \frac{\partial f_{1}{ }^{i} b}{\partial x^{k}}+f_{1}{ }^{i}{ }_{c} f_{3}{ }^{c}{ }_{a b}=0, \\
& \left.f_{1}{ }^{k}{ }_{[d} \frac{\left.\partial f_{3}{ }^{a} b c\right]}{\partial x^{k}}+f_{2}{ }^{a e} f_{4 b c d e}-f_{3}{ }^{a}{ }_{e[b} f_{3}{ }^{e} c d\right] \\
& f_{1}{ }^{k}{ }_{[a} \frac{\partial f_{4 b c d e]}}{\partial x^{k}}+f_{3}{ }^{f}{ }_{[a b} f_{4 c d e] f}=0,
\end{aligned}
$$

where $\left[\begin{array}{llll}b & c & d & \cdots\end{array}\right]$ is a skewsymmetrization with respect to indices $b, c, d, \cdots$, etc.

\subsection{Lie algebroid up to homotopy}

In this section we study an algebraic structure associated with the QP-structure in 3.1 and 3.2.

Definition 3.3 Let $Q=\theta_{2}+\theta_{13}+\theta_{4}$ be a $Q$-structure on $T^{*}[3] E[1]$, where $\left(\theta_{2}, \theta_{13}, \theta_{4}\right)$ is the unique decomposition of $\Theta$. We call the quadruple $\left(E ; \theta_{2}, \theta_{13}, \theta_{4}\right)$ a Lie algebroid up to homotopy, in shorthand, Lie algebroid u.t.h.

We should study the algebraic properties of the Lie algebroid up to homotopy. Let us define a bracket product by

$$
\left[e_{1}, e_{2}\right]:=\left\{\left\{\theta_{13}, e_{1}\right\}, e_{2}\right\}
$$

where $e_{1}, e_{2} \in \Gamma E$. By the bidegree counting, $\Gamma E$ is closed under this bracket. The bracket is not necessarily a Lie bracket, but it is still skewsymmetric:

$$
\begin{aligned}
{\left[e_{1}, e_{2}\right] } & =\left\{\left\{\theta_{13}, e_{1}\right\}, e_{2}\right\} \\
& =\left\{\theta_{13},\left\{e_{1}, e_{2}\right\}\right\}+\left\{e_{1},\left\{\theta_{13}, e_{2}\right\}\right\} \\
& =-\left\{\left\{\theta_{13}, e_{2}\right\}, e_{1}\right\}=-\left[e_{2}, e_{1}\right]
\end{aligned}
$$


where $\left\{e_{1}, e_{2}\right\}=0$ is used. A bundle map $\rho: E \rightarrow T M$ which is called an anchor map is defined by the following identity:

$$
\rho(e)(f):=\left\{\left\{\theta_{13}, e\right\}, f\right\}
$$

where $f \in C^{\infty}(M)$. The bracket and the anchor map satisfy the algebroid conditions (A0) and (A1) below:

(A0) $\rho\left[e_{1}, e_{2}\right]=\left[\rho\left(e_{1}\right), \rho\left(e_{2}\right)\right]$,

(A1) $\left[e_{1}, f e_{2}\right]=f\left[e_{1}, e_{2}\right]+\rho\left(e_{1}\right)(f) e_{2}$,

where the bracket $\left[\rho\left(e_{1}\right), \rho\left(e_{2}\right)\right]$ is the usual Lie bracket on $\Gamma T M$. The bracket (3.16) does not satisfy the Jacobi identity in general. So we should study its Jacobi anomaly, which characterizes the algebraic structure of the Lie algebroid u.t.h. The structures $\theta_{13}, \theta_{2}$ and $\theta_{4}$ define the three operations:

- $\delta(-):=\left\{\theta_{13},-\right\} ;$ a de Rham type derivation on $\Gamma \wedge^{*} E^{*}$,

- $\left(\alpha_{1}, \alpha_{2}\right):=\left\{\left\{\theta_{2}, \alpha_{1}\right\}, \alpha_{2}\right\}$; a symmetric pairing on $E^{*}$, where $\alpha_{1}, \alpha_{2} \in \Gamma E^{*}$,

- $\Omega\left(e_{1}, e_{2}, e_{3}, e_{4}\right):=\left\{\left\{\left\{\left\{\left\{\theta_{4}, e_{1}\right\}, e_{2}\right\}, e_{3}\right\}, e_{4}\right\} ;\right.$ a 4 -form on $E$.

Remark that $\delta \delta \neq 0$ in general. Because the degree of the pairing is -2 , it is $C^{\infty}(M)$-valued. The pairing induces a symmetric bundle map $\partial: E^{*} \rightarrow E$ which is defined by the equation, $\left(\alpha_{1}, \alpha_{2}\right)=\left\langle\partial \alpha_{1}, \alpha_{2}\right\rangle$, where $\langle-,-\rangle$ is the canonical pairing of the duality of $E$ and $E^{*}$. Since $\langle\alpha, e\rangle=\{\alpha, e\}$, we have

$$
\partial \alpha=-\left\{\theta_{2}, \alpha\right\}
$$

By direct computation, we obtain

$$
\frac{1}{2}\left\{\left\{\left\{\left\{\theta_{13}, \theta_{13}\right\}, e_{1}\right\}, e_{2}\right\}, e_{3}\right\}=\left[\left[e_{1}, e_{2}\right], e_{3}\right]+(\text { cyclic permutations })
$$

and

$$
\left\{\left\{\left\{\left\{\theta_{2}, \theta_{4}\right\}, e_{1}\right\}, e_{2}\right\}, e_{3}\right\}=-\partial \Omega\left(e_{1}, e_{2}, e_{3}\right)
$$

From Eq. (3.9), we get an explicit formula of the Jacobi anomaly,

(A2) $\left[\left[e_{1}, e_{2}\right], e_{3}\right]+($ cyclic permutations $)=\partial \Omega\left(e_{1}, e_{2}, e_{3}\right)$. 
In a similar way, we obtain the following identities:

(A3) $\rho \partial=0$,

(A4) $\rho(e)\left(\alpha_{1}, \alpha_{2}\right)=\left(\mathcal{L}_{e} \alpha_{1}, \alpha_{2}\right)+\left(\alpha_{1}, \mathcal{L}_{e} \alpha_{2}\right)$,

(A5) $\delta \Omega=0$,

where $\mathcal{L}_{e}(-):=\left\{\left\{\theta_{13}, e\right\},-\right\}$ is the Lie type derivation which acts on $E^{*}$. Axioms (A3) and (A4) are induced from Eq. (3.8) and (A5) is from Eq. (3.10).

The fundamental relations (3.11)-(3.15) correspond to Axioms (A1)-(A5)e. Thus, the notion of the Lie algebroid up to homotopy is characterized by the algebraic properties (A1)(A5). One concludes that

The classical algebra associated with the QP-manifold $\left(T^{*}[3] E[1], \Theta\right)$ is the space of sections of the vector bundle $E$ with the operations $([\cdot, \cdot], \rho, \partial, \Omega)$ satisfying $(A 1)-(A 5)$.

In the next section, we will study some special examples of Lie algebroid u.t.h.s.

Remark 3.4 If the pairing is nondegenerate, then the bundle map $\partial$ is bijective and then from (A3) we have $\rho=0$.

Remark 3.5 (Higher Courant-Dorfman brackets) We define a bracket on $C^{\infty}(\mathcal{M})$ by

$$
[-,-]_{C D}:=\{\{\Theta,-\},-\}
$$

which is called a Courant-Dorfman (CD) bracket. It is well-known that [, $]_{C D}$ is a Loday bracket $([20]$ ). Since the degree of the CD-bracket is -2 , the total space of degree $i \leq 2$,

$$
C^{2}(\mathcal{M}) \oplus C^{1}(\mathcal{M}) \oplus C^{0}(M)
$$

is closed under the CD-bracket, in particular, the top space $C^{2}(\mathcal{M})=\Gamma\left(E \oplus \wedge^{2} E^{*}\right)$ is a subalgebra. If $\theta_{2}=0$, the CD-bracket on $E \oplus \wedge^{2} E^{*}$ has the following form,

$$
\left[e_{1}+\beta_{1}, e_{2}+\beta_{2}\right]_{C D}=\left[e_{1}, e_{2}\right]+\mathcal{L}_{e_{1}} \beta_{2}-i_{e_{2}} \delta \beta_{1}+\Omega\left(e_{1}, e_{2}\right)
$$

where $\beta_{1}, \beta_{2} \in \Gamma \wedge^{2} E^{*}$. This CD-bracket is regarded as a higher analogue of Courant-Dofman's original bracket (cf. [13]). We refer the reader to Hagiwara [21] and Sheng [22] for the detailed study of the higher CD-brackets.

$\overline{{ }^{e} \text { Actually, the axiom (A0) depends on (A1) }}$ and (A2). 


\section{Examples and twisting transformations}

\subsection{The cases of $\theta_{2}=\theta_{4}=0$}

In this case, the bracket (3.16) satisfies (A0), (A1) and the Jacobi identity. Therefore, the bundle $E \rightarrow M$ becomes a Lie algebroid:

Definition 4.1 (23]) A Lie algebroid over a manifold $M$ is a vector bundle $E \rightarrow M$ with a Lie algebra structure on the space of the sections $\Gamma(E)$ defined by the bracket $\left[e_{1}, e_{2}\right]$ for $e_{1}, e_{2} \in \Gamma(E)$ and an anchor map $\rho: E \rightarrow T M$ satisfying (AO) and (A1) above.

We take $\left\{e_{a}\right\}$ as a local basis of $\Gamma E$ and let a local expression of an anchor map be $\rho\left(e_{a}\right)=$ $f^{i}{ }_{1 a}(x) \frac{\partial}{\partial x^{i}}$ and a Lie bracket be $\left[e_{b}, e_{c}\right]=f_{3}{ }^{a} b c(x) e_{a}$. The Q-structure $\Theta$ associated with the Lie algebroid $E$ is defined as a function on $T^{*}[3] E[1]$,

$$
\Theta:=\theta_{13}:=f_{1}^{i}{ }_{a}(x) \xi_{i} q^{a}+\frac{1}{2} f_{3}^{a} b c(x) p_{a} q^{b} q^{c}
$$

which is globally well-defined. Conversely, if we consider $\Theta:=\theta_{13}$, the classical master equation induces the Lie algebroid structure on $E$.

Let us consider the case that the bundle is a vector space on a point. A Lie algebroid over a point $\mathfrak{g} \rightarrow\{p t\}$ is a Lie algebra $\mathfrak{g}$. The P-manifold over $\mathfrak{g} \rightarrow\{p t\}$ is isomorphic to $\mathfrak{g}^{*}[2] \oplus \mathfrak{g}[1]$ and the structure sheaf is the polynomial algebra over $\mathfrak{g}[2] \oplus \mathfrak{g}^{*}[1]$,

$$
C^{\infty}(\mathcal{M})=S(\mathfrak{g}) \otimes \bigwedge \mathfrak{g}^{*}
$$

The bidegree is defined by the natural manner,

$$
C^{2 i, j}(\mathcal{M})=S^{i}(\mathfrak{g}) \otimes \bigwedge^{j} \mathfrak{g}^{*}
$$

The Q-structure associated with the Lie bracket on $\mathfrak{g}$ is

$$
\theta_{13}=\frac{1}{2} f_{b c}^{a} p_{a} q^{b} q^{c} \cong \frac{1}{2} f_{b c}^{a} p_{a} \otimes\left(q^{b} \wedge q^{c}\right),
$$

where $p . \in \mathfrak{g}, q . \in \mathfrak{g}^{*}$ and $f_{b c}^{a}$ is the structure constant of the Lie algebra. 


\subsection{The cases of $\theta_{2} \neq 0$ and $\theta_{4}=0$}

In this case, the bracket induced by $\theta_{13}$ still satisfies the Jacobi identity.

We assume that $\mathfrak{g}$ is semi-simple. Then the dual space $\mathfrak{g}^{*}$ has a metric, $(\cdot, \cdot)_{K^{-1}}$, which is the inverse of the Killing form on $\mathfrak{g}$. The metric inherits the following invariant condition from the Killing form:

$$
\left(\mathcal{L}_{p} q_{1}, q_{2}\right)_{K^{-1}}+\left(q_{1}, \mathcal{L}_{p} q_{2}\right)_{K^{-1}}=0
$$

where $\mathcal{L}_{p}(-)$ is the canonical coadjoint action of $\mathfrak{g}$ to $\mathfrak{g}^{*}$. Eq. (4.18) is a linear version of (A4). Thus, we obtain a Q-structure,

$$
\Theta:=k^{a b} p_{a} p_{b}+\frac{1}{2} f_{b c}^{a} p_{a} q^{b} q^{c},
$$

where $k^{a b} p_{a} p_{b}:=(\cdot, \cdot)_{K^{-1}}$.

\subsection{Non Lie algebra example}

We consider the cases that the Jacobi identity is broken. Let $\left(\mathfrak{g},[\cdot, \cdot],(\cdot, \cdot)_{K}\right)$ be a vector space (not necessarily Lie algebra) equipped with a skewsymmetric bracket $[\cdot, \cdot]$ and an invariant metric $(\cdot, \cdot)_{K}$. The metric induces a bijection $K: \mathfrak{g} \rightarrow \mathfrak{g}^{*}$ which is defined by the identity,

$$
\left(p_{1}, p_{2}\right)_{K}=\left\langle K p_{1}, p_{2}\right\rangle
$$

We define a map from $\mathfrak{g}^{*}$ to $\mathfrak{g}$ by $\partial:=K^{-1}$ and define a 4 -form by,

$$
\Omega\left(p_{1}, p_{2}, p_{3}, p_{4}\right):=\left(\left[\left[p_{1}, p_{2}\right], p_{3}\right]+\text { cyclic permutations, } p_{4}\right)_{K} .
$$

Remark 4.2 The 4-form above is considered to be a higher analogue of the Cartan 3-form $\left(\left[p_{1}, p_{2}\right], p_{3}\right)_{K}$.

Axioms (A0)-(A4) obviously hold on $\mathfrak{g}$. We check (A5). It suffices to show (3.10). Let us denote by $\left\{-, p_{1}, p_{2}, \ldots, p_{n}\right\}$ the $n$-fold bracket $\left\{\ldots\left\{\left\{-, p_{1}\right\}, p_{2}\right\}, \ldots, p_{n}\right\}$. We already have (3.8) and (3.9). From $\left\{\theta_{13},\left\{\theta_{13}, \theta_{13}\right\}\right\}=0$ and (3.9) , we have $\left\{\theta_{13},\left\{\theta_{2}, \theta_{4}\right\}\right\}=0$. Since $\left\{\theta_{13}, \theta_{2}\right\}=0$, this is equal to $\left\{\theta_{2},\left\{\theta_{3}, \theta_{4}\right\}\right\}=0$ up to sign. This gives $\left\{\left\{\theta_{2},\left\{\theta_{3}, \theta_{4}\right\}\right\}, p_{1}, \ldots, p_{5}\right\}=0$ for any $p_{1}, \ldots, p_{5}$. From $\left\{\theta_{2}, p\right\}=0$, we have

$$
\left\{\theta_{2},\left\{\left\{\theta_{3}, \theta_{4}\right\}, p_{1}, \ldots, p_{5}\right\}\right\}=0 .
$$


Since $K^{-1}=-\left\{\theta_{2},-\right\}$ is bijective, we get

$$
\left\{\left\{\theta_{3}, \theta_{4}\right\}, p_{1}, \ldots, p_{5}\right\}=0
$$

which yields the desired relation $\left\{\theta_{3}, \theta_{4}\right\}=0$.

Proposition 4.3 The triple $(\mathfrak{g}, \partial, \Omega)$ is a Lie algebra(oid) up to homotopy.

\subsection{Twisting by 3 -form and the cases of $\theta_{2}=0$ and $\theta_{4} \neq 0$}

We introduce the notion of twisting transformation by 3-form before studying the cases of $\theta_{2}=0$. Given a Q-structure $\Theta$ and a 3-form $\phi \in C^{0,3}(\mathcal{M})$, there exists the second Q-structure which is defined by the canonical transformation,

$$
\Theta^{\phi}:=\exp \left(X_{\phi}\right)(\Theta)
$$

where $X_{\phi}:=\{\phi,-\}$ is the Hamiltonian vector field of $\phi$. The transformation (4.20) is called a twisting by 3-form, or simply twisting. By a direct computation, we obtain

$$
\begin{aligned}
\theta_{2}^{\phi} & =\theta_{2}, \\
\theta_{13}^{\phi} & =\theta_{13}-\left\{\theta_{2}, \phi\right\}, \\
\theta_{4}^{\phi} & =\theta_{4}-\left\{\theta_{13}, \phi\right\}+\frac{1}{2}\left\{\left\{\theta_{2}, \phi\right\}, \phi\right\},
\end{aligned}
$$

where $\Theta^{\phi}=\theta_{2}^{\phi}+\theta_{13}^{\phi}+\theta_{4}^{\phi}$ and $X_{\phi}^{i \geq 3}(\Theta)=0$. The twisting by 3 -form defines an equivalence relation on the Q-structures.

We notice that $\theta_{2}$ is an invariant for the twisting. If $\theta_{2}=0$, then $\theta_{13}$ is an invariant and

$$
\theta_{4}^{\phi}=\theta_{4}-\delta \phi
$$

where $\delta \phi=\left\{\theta_{13}, \phi\right\}$. This leads us

Proposition 4.4 The class of Q-structures which have no $\theta_{2}$ is classified into $H_{d R}^{4}\left(\bigwedge^{*} E^{*}, \delta\right)$ by the twisting by 3-form. 


\section{AKSZ Construction of Topological Field Theory in 4 Dimensions}

\subsection{General Theory}

In this section, we consider the AKSZ construction of a topological field theory in 4 dimensions.

For a graded manifold $\mathcal{N}$, let $\left.\mathcal{N}\right|_{0}$ be the degree zero part.

Let $X$ be a manifold in 4 dimensions and $M$ be a manifold in $d$ dimensions. Let $(\mathcal{X}, D)$ be a differential graded $(\mathrm{dg})$ manifold $\mathcal{X}$ with a $D$-invariant nondegenerate measure $\mu$, such that $\left.\mathcal{X}\right|_{0}=X$, where $D$ is a differential on $\mathcal{X} .(\mathcal{M}, \omega, \Theta)$ is a QP-manifold of degree 3 and $\left.\mathcal{M}\right|_{0}=M$. A degree $\operatorname{deg}(-)$ on $\mathcal{X}$ is called the form degree and a degree $\operatorname{gh}(-)$ on $\mathcal{M}$ is called the ghost number ff $\operatorname{Let} \operatorname{Map}(\mathcal{X}, \mathcal{M})$ be a space of smooth maps from $\mathcal{X}$ to M. $|-|=\operatorname{deg}(-)+\operatorname{gh}(-)$ is the degree on $\operatorname{Map}(\mathcal{X}, \mathcal{M})$ and called the total degree. $\mathrm{A}$ $\mathrm{QP}$-structure on $\operatorname{Map}(\mathcal{X}, \mathcal{M})$ is constructed from the above data.

Since $\operatorname{Diff}(\mathcal{X}) \times \operatorname{Diff}(\mathcal{M})$ naturally acts on $\operatorname{Map}(\mathcal{X}, \mathcal{M}), D$ and $Q$ induce homological vector fields on $\operatorname{Map}(\mathcal{X}, \mathcal{M}), \hat{D}$ and $\check{Q}$.

Two maps are introduced. An evaluation map ev $: \mathcal{X} \times \mathcal{M}^{\mathcal{X}} \longrightarrow \mathcal{M}$ is defined as

$$
\mathrm{ev}:(z, \Phi) \longmapsto \Phi(z)
$$

where $z \in \mathcal{X}$ and $\Phi \in \mathcal{M}^{\mathcal{X}}$.

A chain map $\mu_{*}: \Omega^{\bullet}(\mathcal{X} \times \mathcal{M}) \longrightarrow \Omega^{\bullet}(\mathcal{M})$ is defined as $\mu_{*} F=\int_{\mathcal{X}} \mu F$ where $F \in \Omega^{\bullet}(\mathcal{X} \times \mathcal{M})$

and $\int_{\mathcal{X}} \mu$ is an integration on $\mathcal{X}$ by the $D$-invariant measure $\mu$. It is an usual integral for the even degree parts and the Berezin integral for the odd degree parts.

A (classical) $\mathrm{P}$-structure on $\operatorname{Map}(\mathcal{X}, \mathcal{M})$ is defined as follows:

Definition 5.1 For a graded symplectic form $\omega$ on $\mathcal{M}$, a graded symplectic form $\boldsymbol{\omega}$ on $\operatorname{Map}(\mathcal{X}, \mathcal{M})$ is defined as $\boldsymbol{\omega}:=\mu_{*} \mathrm{ev}^{*} \omega$.

We can confirm that $\boldsymbol{\omega}$ satisfies the definition of a graded symplectic form because $\mu_{*} \mathrm{ev}^{*}$ preserves nondegeneracy and closedness. Thus $\boldsymbol{\omega}$ is a $\mathrm{P}$-structure on $\operatorname{Map}(\mathcal{X}, \mathcal{M})$ and induces $\bar{f}$ The ghost number $\operatorname{gh}(-)$ is the degree $|-|$ on $\mathcal{M}$ in section 2. 
a graded Poisson bracket $\{-,-\}$ on $\operatorname{Map}(\mathcal{X}, \mathcal{M})$. Since $\left|\mu_{*} \mathrm{ev}^{*}\right|=-4,|\boldsymbol{\omega}|=-1$ and $\{-,-\}$ on $\operatorname{Map}(\mathcal{X}, \mathcal{M})$ has degree 1 and an odd Poisson bracket.

Next we define a $\mathrm{Q}$-structure $S$ on $\operatorname{Map}(\mathcal{X}, \mathcal{M}) . S$ is called a $B V$ action and consists of two parts $S=S_{0}+S_{1}$. $S_{0}$ is constructed as follows: Let $\omega$ be the odd symplectic form on $\mathcal{M}$. We take a fundamental form $\vartheta$ such that $\omega=-d \vartheta$ and define $S_{0}:=\iota_{\hat{D}} \mu_{*} \mathrm{ev}^{*} \vartheta .\left|S_{0}\right|=0$ because $\mu_{*} \mathrm{ev}^{*}$ has degree -4 . $S_{1}$ is constructed as follows: We take a Q-structure $\Theta$ on $\mathcal{M}$ and define $S_{1}:=\mu_{*} \mathrm{ev}^{*} \Theta . S_{1}$ also has degree 0 .

We can prove that $S$ is a $\mathrm{Q}$-structure on $\operatorname{Map}(\mathcal{X}, \mathcal{M})$, since

$$
\{\Theta, \Theta\}=0, \Longleftrightarrow\{S, S\}=0
$$

from the definition of $S_{0}$ and $S_{1}$.

A quantum version is

$$
\Delta\left(e^{\frac{i}{\hbar} \Theta}\right)=0 \Longleftrightarrow \hat{\Delta}\left(e^{\frac{i}{\hbar} S}\right)=0,
$$

where $\hat{\Delta}$ is an odd Laplace operator on $\operatorname{Map}(\mathcal{X}, \mathcal{M})$. The infinitesimal form of the right hand side in (5.22) is $\{S, S\}-2 i \hbar \hat{\Delta} S=0$, which is called a quantum master equation. $\left.\right|^{g}$

The following theorem has been confirmed [3]:

Theorem 5.2 If $\mathcal{X}$ is a dg manifold and $\mathcal{M}$ is a $Q P$-manifold, the graded manifold $\operatorname{Map}(\mathcal{X}, \mathcal{M})$ has a QP-structure.

Definition 5.3 A topological field theory in 4 dimensions is a triple $(\mathcal{X}, \mathcal{M}, S)$, where $\mathcal{X}$ is a dg manifold with $\left.\operatorname{dim} \mathcal{X}\right|_{0}=4, \mathcal{M}$ is a $Q P$-manifold with the degree 3 , and $S$ is a $B V$ action with the total degree 0.

In order to interpret this theory as a 'physical' topological field theory, we must take $\mathcal{X}=T[1] X$. Then we can confirm that a $\mathrm{QP}$-structure on $\operatorname{Map}(\mathcal{X}, \mathcal{M})$ is equivalent to the AKSZ formulation of a topological field theory [4][10]. We set $\mathcal{X}=T[1] X$ from now.

In 'physics', a quantum field theory is constructed by quantizing a classical field theory. First we consider a Q-structure $\{\cdot, \cdot\}$ and a classical P-structure $S$ such that

$$
\{S, S\}=0
$$

${ }^{g}$ Discussion for an odd Laplace operator is too naive. In general, the quantum master equation has an obstruction expressed by the modular class [24]. We must regularize an odd Laplace operator and a quantum BV action. 
Next we define a quantum P-structure $\hat{\Delta}$ and confirm that

$$
\tilde{\Delta}\left(e^{\frac{i}{\hbar} S}\right)=0
$$

Finally we calculate a partition function

$$
Z=\int_{\mathcal{L}} e^{\frac{i}{\hbar} S}
$$

on a Lagrangian submanifold $\mathcal{L} \subset \operatorname{Map}(\mathcal{X}, \mathcal{M})$. Quantization is not discussed in this paper.

\subsection{Local Coordinate Expression and Examples}

A general theory in the previous subsection is applied to the local coordinate expression in section 3.1 and a known topological field theory in 4 dimensions is obtained as a special case and a new nontrivial topological field theory is constructed. Let us take a manifold $X$ in 4 dimensions and a manifold $M$ in $d$ dimensions. Let $E[1]$ is a graded vector bundle on $M$. We take $\mathcal{X}=T[1] X$ and $\mathcal{M}=T^{*}[3] E[1]$.

Let $\left(\sigma^{\mu}, \theta^{\mu}\right)$ be a local coordinate on $T[1] X . \sigma^{\mu}$ is a local coordinate on the base manifold $X$ and $\theta^{\mu}$ is one on the fiber of $T[1] X$, respectively. Let $\boldsymbol{x}^{i}$ be a smooth map $\boldsymbol{x}^{i}: X \longrightarrow M$ and $\boldsymbol{\xi}_{i}$ be a section of $T^{*}[1] X \otimes \boldsymbol{x}^{*}\left(T^{*}[3] M\right), \boldsymbol{q}^{a}$ be a section of $T^{*}[1] X \otimes \boldsymbol{x}^{*}(E[1])$ and $\boldsymbol{p}_{a}$ be a section of $T^{*}[1] X \otimes \boldsymbol{x}^{*}\left(T^{*}[3] E \boldsymbol{x}[1]\right)$. These are called superfields. The exterior derivative $d$ is taken as a differential $D$ on $X$. From $d$, a differential $\boldsymbol{d}=\theta^{\mu} \frac{\partial}{\partial \sigma^{\mu}}$ on $\mathcal{X}$ is induced.

Then a BV action $S$ has the following expression:

$$
\begin{aligned}
S & =S_{0}+S_{1}, \\
S_{0} & =\int_{\mathcal{X}} \mu\left(\boldsymbol{\xi}_{i} \boldsymbol{d} \boldsymbol{x}^{i}-\boldsymbol{p}_{a} \boldsymbol{d} \boldsymbol{q}^{a}\right), \\
S_{1} & =\int_{\mathcal{X}} \mu\left(f_{1}{ }_{a}(\boldsymbol{x}) \boldsymbol{\xi}_{i} \boldsymbol{q}^{a}+\frac{1}{2} f_{2}^{a b}(\boldsymbol{x}) \boldsymbol{p}_{a} \boldsymbol{p}_{b}+\frac{1}{2} f_{3}{ }_{b c}(\boldsymbol{x}) \boldsymbol{p}_{a} \boldsymbol{q}^{b} \boldsymbol{q}^{c}+\frac{1}{4 !} f_{4 a b c d}(\boldsymbol{x}) \boldsymbol{q}^{a} \boldsymbol{q}^{b} \boldsymbol{q}^{c} \boldsymbol{q}^{d}\right) .
\end{aligned}
$$

Nonabelian BF theory. Let $\Theta$ be a Q-structure (4.17) for a Lie algebra $\mathfrak{g} \cdot \boldsymbol{\xi}_{i} \boldsymbol{d} \boldsymbol{x}^{i}=0$ since $M=\{p t\}$. If we define a curvature $\boldsymbol{F}^{a}=\boldsymbol{d} \boldsymbol{q}^{a}-\frac{1}{2} f^{a}{ }_{b c} \boldsymbol{q}^{b} \boldsymbol{q}^{c}$, a Q-structure is

$$
S=\int_{\mathcal{X}} \mu\left(-\boldsymbol{p}_{a} \boldsymbol{F}^{a}\right)
$$

which is equivalent to a $\mathrm{BV}$ formalism for a nonabelian $\mathrm{BF}$ theory in 4 dimensions. 
Topological Yang-Mills Theory. We take a nondegenerate Killing form $(\cdot, \cdot)_{K}$ for a Lie algebra $\mathfrak{g}$ and consider the Q-structure (4.19). A topological field theory constructed from (4.19) is

$$
S=\int_{\mathcal{X}} \mu\left(-\boldsymbol{p}_{a} \boldsymbol{F}^{a}+k^{a b} \boldsymbol{p}_{a} \boldsymbol{p}_{b}\right) .
$$

This is equivalent to a topological Yang-Mills theory,

$$
S=-\frac{1}{4} \int_{\mathcal{X}} \mu k_{a b} \boldsymbol{F}^{a} \boldsymbol{F}^{b},
$$

if we delete $\boldsymbol{p}_{a}$ by the equations of motion.

Nonassociative BF Theory. Let us take a non Lie algebra $\left(\mathfrak{g},[\cdot, \cdot],(\cdot, \cdot)_{K}\right)$ in section 4.3. If we take $M=\{p t\}$ and $\mathcal{M}=\mathfrak{g}^{*}[2] \oplus \mathfrak{g}[1],\left(\mathfrak{g},[\cdot, \cdot],(\cdot, \cdot)_{K}\right)$ leads a QP-structure with degree 3. In the canonical basis, it is expressed as

$$
\begin{aligned}
& f_{1{ }^{i} a}(x)=0, \quad f_{2}^{a b}(x)=K^{a b}, \\
& f_{3 b c}^{a}(x)=f_{b c}^{a}, \quad f_{4 a b c d}(x)=K_{a e}^{-1} f_{f[b}^{e} f_{c d]}^{f},
\end{aligned}
$$

where $K^{a b}=\left(p_{a}, p_{b}\right)$ is nondegenerate and $\left[p_{a}, p_{b}\right]=f_{a b}^{c} p_{c}$ is a nonassociative bracket and does not satisfy the Jacobi identity. The AKSZ construction derives a new nontrivial topological field theory in 4 dimensions. A BV action $S$ has the following expression:

$$
\begin{aligned}
S & =\int_{\mathcal{X}} \mu\left(-\boldsymbol{p}_{a} \boldsymbol{d} \boldsymbol{q}^{a}+\frac{1}{2} K^{a b} \boldsymbol{p}_{a} \boldsymbol{p}_{b}+\frac{1}{2} f_{b c}^{a} \boldsymbol{p}_{a} \boldsymbol{q}^{b} \boldsymbol{q}^{c}+\frac{1}{4 !} K_{a e}^{-1} f_{f[b}^{e} f_{c d]} \boldsymbol{q}^{a} \boldsymbol{q}^{b} \boldsymbol{q}^{c} \boldsymbol{q}^{d}\right) \\
& =-\frac{1}{4} \int_{\mathcal{X}} \mu\left(K_{a b} \boldsymbol{F}^{a} \boldsymbol{F}^{b}+\frac{1}{3 !} K_{a e}^{-1} f_{f[b}^{e} f_{c d]}^{f} \boldsymbol{q}^{a} \boldsymbol{q}^{b} \boldsymbol{q}^{c} \boldsymbol{q}^{d}\right) .
\end{aligned}
$$

It is easily confirmed that $\{S, S\}=0$.

Topological 3-brane on $\operatorname{Spin}(7)$-structure. Let $(M, \Omega)$ be an 8-dimensional $\operatorname{Spin}(7)$ manifold. Here $\Omega$ is a $\operatorname{Spin}(7) 4$-form, which satisfies $d \Omega=0$ and the selfdual condition $\Omega=* \Omega$. A $\operatorname{Spin}(7)$ structure is defined as the subgroup of $G L(8)$ to preserve $\Omega$. The Q-structure on $(T M, \Omega)$ is given by

$$
\Theta=\xi_{i} q^{i}+\frac{1}{4 !} \Omega_{i j k l}(x) q^{i} q^{j} q^{k} q^{l}
$$

The BV action $S$ for (5.23) defines the same theory as the topological 3-brane analyzed in [25]. 


\section{Conclusions and Discussion}

We have defined a BV algebra and a QP-structure of degree 3. A QP-structure of degree 3 has been constructed explicitly and a Lie algebroid u.t.h. has been defined as its algebraic and geometric structure. A general theory of the AKSZ construction of a topological field theory has been expressed and a new topological field theory in four dimensions has been constructed from a QP-structure.

Quantization of this theory and analysis of a Lie algebroid u.t.h. will shed light on a super Poisson geometry and a quantum field theory. They are future problems.

\section{Acknowledgements}

The authors would like to thank Klaus Bering, Maxim Grigoriev, Camille Laurent-Gengoux, Yvette Kosmann-Schwarzbach, Kirill Mackenzie, Dmitry Roytenberg, Alexei Sharapov, Thomas Strobl and Theodore Voronov for their comments and discussion. The author (N.I.) would like to thank Maskawa Institute for Science and Culture, Kyoto Sangyo University for hospitality. We would like to thank to referees for their useful advice.

\section{References}

[1] I.A. Batalin and G.A. Vilkovisky, Phys. Lett. B102 (1981) 27;

Phys. Rev. D28 (1983) 2567.

[2] A. S. Schwarz, Commun. Math. Phys. 155 (1993) 249 hep-th/9205088; Commun. Math. Phys. 158 (1993) 373 [hep-th/9210115].

[3] M. Alexandrov, M. Kontsevich, A. Schwartz and O. Zaboronsky, Int. J. Mod. Phys. A 12 (1997) 1405 hep-th/9502010].

[4] A. S. Cattaneo and G. Felder, Lett. Math. Phys. 56, 163 (2001) math.QA/0102108.

[5] J. S. Park, Seoul 2000, Symplectic geometry and mirror symmetry, Singapore. World Scientific, 2001, 311-384, hep-th/0012141. 
[6] P. Severa, Travaux Math. 16 (2005) 121-137, math.SG/0105080.

[7] N. Ikeda, JHEP 0107, 037 (2001), hep-th/0105286].

[8] N. Ikeda and K. I. Izawa, Prog. Theor. Phys. 90 (1993) 237 hep-th/9304012]. For reviews, N. Ikeda, Annals Phys. 235 (1994) 435 [hep-th/9312059].

[9] P. Schaller and T. Strobl, Mod. Phys. Lett. A 9 (1994) 3129 hep-th/9405110. [hep-th/9411163].

[10] N. Ikeda, math.SG/0604157.

[11] M. Kontsevich, Lett. Math. Phys. 66 (2003) 157 [math.QA/9709040].

[12] A. S. Cattaneo and G. Felder, Commun. Math. Phys. 212, 591 (2000) math.QA/9902090.

[13] T. Courant, Trans. A. M. S. 319 (1990) 631. Z. J. Liu, A. Weinstein and P. Xu, math.DG/9611001.

[14] D. Roytenberg, math.DG/9910078; math.QA/0112152; math.SG/0203110.

[15] N. Ikeda, Int. J. Mod. Phys. A 18 (2003) 2689; JHEP 0210 (2002) 076 hep-th/0209042.

[16] C. Hofman and J. S. Park, hep-th/0209148.

[17] D. Roytenberg, Lett. Math. Phys. 79 (2007) 143 hep-th/0608150].

[18] M. Grützmann, arXiv:1005.5680].

[19] H. O. M. Khudaverdian, Commun. Math. Phys. 247 (2004) 353, math.DG/0012256.

[20] Y. Kosmann-Schwarzbach, Lett. Math. Phys. 69, 61 (2004), math.DG/0312524.

[21] Y. Hagiwara, J. Phys. A: Math. Gen. 35 (2002) 1263.

[22] Y. Sheng. arXiv:1003.1350[math.DG]

[23] K. Mackenzie, Lie Groupoids and Lie Algebroids in Differential Geometry, LMS Lecture Note Series 124, Cambridge U. Press, (1987). 
[24] S. L. Lyakhovich and A. A. Sharapov, Nucl. Phys. B 703 (2004) 419 arXiv:hep-th/0407113.

[25] G. Bonelli and M. Zabzine, JHEP 0509 (2005) 015 hep-th/0507051]. 